\title{
VERTICAL CLIMBING LOCOMOTION OF A NEW GECKO ROBOT USING DRY ADHESIVE MATERIAL
}

\author{
Zhiwei Yu, ${ }^{*}$ Bin Yang, ${ }^{*}$ Simon X. Yang, ${ }^{* *}$ and Zhendong Dai ${ }^{*}$
}

\begin{abstract}
Most of Geckos can move freely on a smooth vertical surface. The wonderful ability of locomotion depended on the countless setae under the geckos' foot which have an effect on van der Waals' force between foot and surface. This kind of force belongs to the dry adhesive force. Many scientists had attempted to understand structure-function and had produced several kinds of dry adhesive material. One of the dry adhesive materials with mushroom-shaped adhesive microstructure (MSAMS) had been selected and firstly applied in vertical locomotion of Gecko robot_6 which was a bioinspired gecko robot with four legs. To apply MSAMS in Gecko robot_6 successfully, the adhesive ability of MSAMS was tested including normal force, tangential force, and the peel-off force curve with different thickness substrates of bio-inspired gecko foot. The new bio-inspired gecko foot with MSAMS was designed, and the Gecko robot_6 can climb on a smooth vertical surface (glass) stably using dry adhesive material with MSAMS.
\end{abstract}

\section{Key Words}

Gecko robot, vertical climbing locomotion, dry adhesive material

\section{Introduction}

Most of Geckos can move freely on a smooth vertical surface, their super adhesive ability depends largely on van der Waals forces of the nearly hundred thousand keratinous hairs or setae on the toe [1]-[4]. The dynamics of geckos running vertically had been tested, and Autumn et al. found that differential leg function appeared essential for effective vertical as well as horizontal locomotion [5]. To measure the dynamics of gecko locomotion, Dai et al. developed a force-measuring array to measure 3D reaction forces of gecko [6], and our team had found the dynamics of

* Institute of Bio-inspired Structure and Surface Engineering, College of Astronautics, Nanjing University of Aeronautics and Astronautics, People's Republic of China; e-mail: \{yuzhiwei, zddai\}@nuaa.edu.cn, 1039843345@qq.com

** Advanced Robotics and Intelligent Systems (ARIS) Laboratory, School of Engineering, University of Guelph, Guelph, Ontario, Canada N1G 2W1; e-mail: syang@uoguelph.ca

Recommended by Prof. Anmin Zhu

(DOI: 10.2316/Journal.206.2017.4.206-5054) gecko on horizontal, vertical and ceiling locomotion [7], [8]. Many researchers began to study the gecko adhesive ability, and hoped to produce bio-inspired gecko tape applied in a climbing robot. Sitti and Fearing produced a preliminary nano-hair prototype which showed adhesion close to the predicted values for natural specimens (around $100 \mathrm{nN}$ each) [9]. Aksak et al. developed several new modifications to the tips of the fibre arrays including angled spatula tips for directional adhesion and friction and hierarchical fibres [10]. Das et al. demonstrated the effect of roughness and shearing velocities on the stick-slip friction between tilted polydimethylsiloxane (PDMS) micro-flaps and silica surfaces and the stick-slip friction of gecko-mimetic flaps on smooth and rough surfaces was discussed in [11].

Researchers designed many kinds of climbing robot, such as wheel-legged, pedrail, tank-like and track-wheel climbing robot. Mini-Whegs ${ }^{\mathrm{TM}}$ used two-compound polymer polyvinylsiloxane (PVS), and applied a single motor to drive their multi-spoke wheel-leg appendages for wall climbing locomotion [12]. Wu et al. developed a pedrail robot which was climbing on a vertical wall and a wooden door using polydimethylsiloxane adhesive material [13]. Seo and Sitti developed a prototype of tank-like modulebased climbing robot using a flat dry elastomer adhesive [14]. Koh et al. presented application of a hybrid adhesive for a track-wheel robot which had demonstrated that the functional material structure generates adhesive forces from electrostatic actuation and from flat smooth elastomer effect that enhances each other in a symbiosis manner [15].

Some of climbing robots cannot climb on a $90^{\circ}$ vertical surface. Menon et al. focused on the development of gecko-inspired synthetic dry adhesives for wallclimbing robots. The wireless vehicle, equipped with PDMS treads, was able to climb an acrylic surface sloped at $75^{\circ}$ showing a very reliable behaviour [16], [17]. Unver et al. developed a gecko-inspired climbing robot which walks and climbs up to $85^{\circ}$ acrylic surfaces [18]. He et al. developed a novel wet adhesion pad for wall-climbing robots which can climb up to more than $80^{\circ}$ sloped surface and stick to the vertical surface statically [19]. Chen et al. developed a robot inspired by gecko locomotion based on an electrostatic adhesion mechanism, 


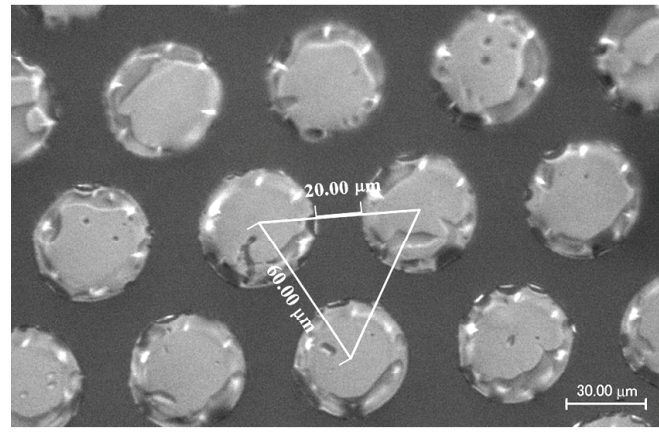

(a)

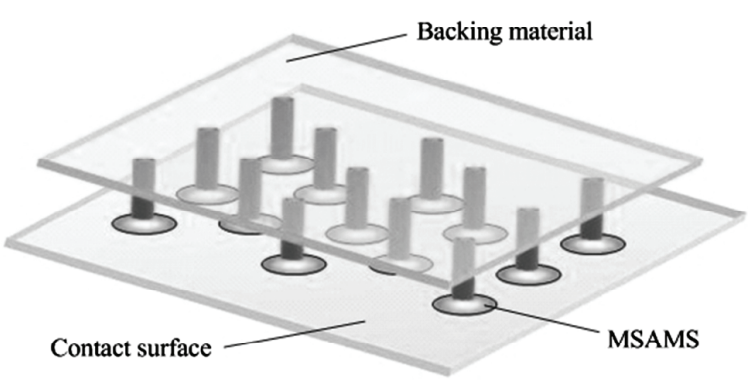

(b)

Figure 1. (a) Geometry sizes of MSAMS and (b) the model of MSAMS.

and the robot climbed on a vertical glass surface with a slope angle not exceeding $75^{\circ}[20]$. Birkmeyer et al. presented a climbing autonomous sprawled hexapod which was equipped with a remote centre-of-motion ankle and gecko-inspired adhesive feet, and it can climb the $70^{\circ}$ incline at $10 \mathrm{~cm} / \mathrm{s}$ velocity [21]. Yue et al. presented a new retractable-claw wheel with six sets of claw mechanism which could climb $40^{\circ}$ sloped terrains [22]. Jaradat et al. presented a portable lightweight climbing robot for a smooth sloping surface, and designed the bio-inspired climbing skills from inchworms [23].

Some of legged climbing robots had been developed. Henrey et al. presented a legged, hexapod robot using dry adhesive footpads which allowed the robot to stick to smooth surfaces [24]. Wang et al. proposed a new dynamic model and designed a climbing prototype robot which sticks to the surface by claw [25]. Kim et al. described the design and control of a Stickybot which can climb smooth vertical surfaces using directional adhesive materials [26]. Because Stickybot's feet were driven by four motors, and its legs were only driven by eight motors, it had less degree of freedoms in locomotion.

After studying a survey of wall-climbing robots, researchers expected to develop robots of high ability by mimicking the characteristics of animals which can climb up walls, like geckos. As no large-sized mechanisms are necessary for adhesion with bio-inspired approaches, Nansai and Mohan said that most of climbing robots were designed to be small and light weight [27].

Generally speaking, the nanotechnology-based materials for adhesion will be a renewed interest in the field of bio-inspired wall-climbing robots. Therefore, we will use the dry adhesive material on a bio-inspired legged climbing robot which mimicking the gecko, and develop the vertical locomotion ability with a higher payload capacity. During the experiment of the vertical locomotion, some approaches in tracking control were referenced from these papers [28], [29].

This paper was organized in the following sequence. Section 2 presents the structure of dry adhesive material with mushroom-shaped adhesive microstructure (MSAMS) and shows the experimental methods during the testing. Section 3 shows the results of the testing data of adhesive properties. Section 4 discusses the testing data results, and gives the suggestion to guide the design of the bio-inspired gecko foot. Section 5 shows the Gecko robot_6 and the structure of bio-inspired gecko foot which applied dry adhesive material with MSAMS in smooth vertical surface (glass) locomotion. Finally, conclusions and future work are reported in Section 6.

\section{Materials and Methods}

Dai from Institute of Bio-inspired Structure and Surface Engineering (IBSS) has the research cooperation with Gorb who has provided dry adhesive material with the MSAMS which was made of PVS. Gorb et al. had shown the geometry structure of MSAMS in his papers [30]. When we start to apply this kind of dry adhesive material in the gecko robot, we observed the geometry structure of MSAMS using an optical microscope, and measured the geometry sizes of mushroom-shaped microstructure. Figure 1 shows the picture of the MSAMS. The diameter of mushroom-shaped microstructure was about $40 \mu \mathrm{m}$, and the central distance between the two mushroom-shaped microstructures was about $60 \mu \mathrm{m}$. Most of the head mushroom-shaped microstructures were like disc shaped. The distribution of three mushroom-shaped microstructures was like the equilateral triangle. After the observation of MSAMS, we designed the model of MSAMS as shown in Fig. 1(b). The dry adhesive material was composed of MSAMS and back material. When the dry adhesive material was used, the head of MSAMS will contact the smooth surface such as glass and mirror. We selected dry adhesive material with the sizes of $20 \mathrm{~mm} \times 20 \mathrm{~mm}$, which will be tested in the following experiments.

Gorb et al. had analysed the different factors influencing adhesion, such as the presence of fluid in contact, sample structure, substrate roughness and geometry [31]. The adhesion of MSAMS had been measured when MSAMS was completely submerged under water. Pull-off forces with different pull-off velocities were measured under atmospheric pressure and reduced pressure [32].

Gorb had tested the properties of dry adhesive materials, and helped us to understand the ability of adhesion. But he did not apply in the bio-inspired four legs gecko robot well in vertical locomotion control. When we used the dry adhesive material, we should combine it with the 


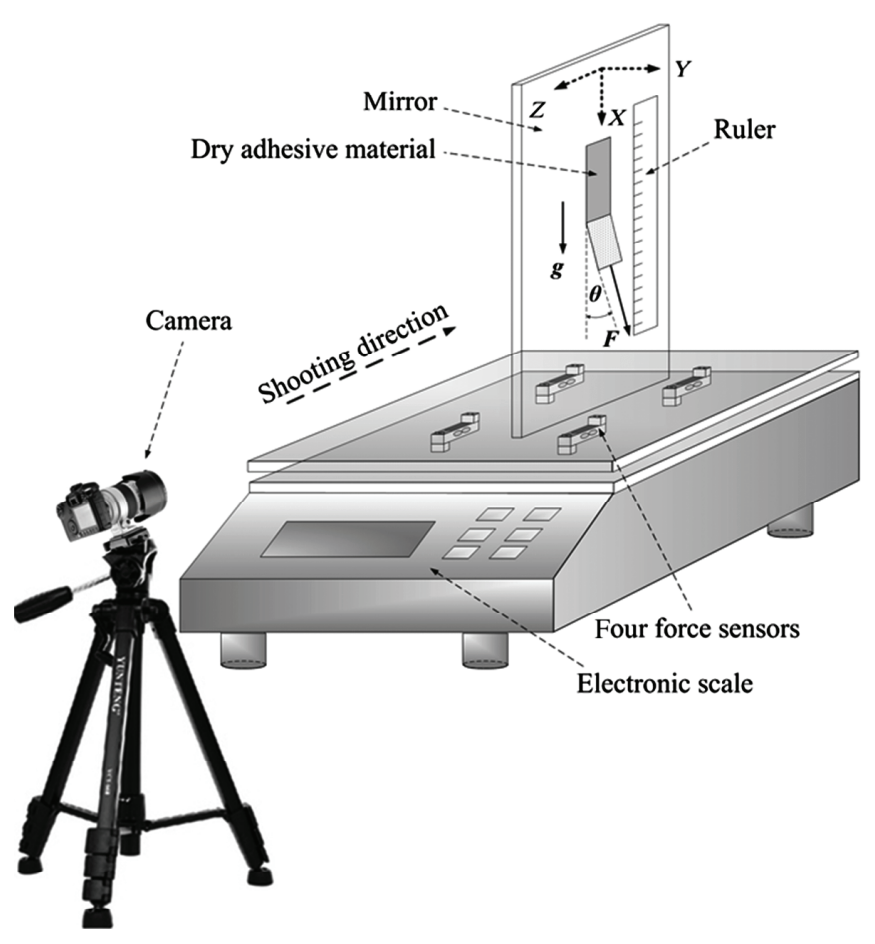

Figure 2. Equipment of the experiment.

design of gecko robot's foot, and test the properties of dry adhesive materials much more in detail. It will contribute to design the gecko robot's foot perfectly.

In this paper, we will analyse different factors which influence adhesion performance. The test of experiments as follows: (1) the relationship between the sizes of contact area and normal force, (2) the relationship between the sizes of contact area and tangential force, and (3) the relationship between the peel-off forces and angles $(\theta)$ in the peel-off force curve under using different thickness substrates of gecko robot's foot.

To measure the peel-off tangential force of dry adhesive material, we carried out the experiments using an electronic scale which had four one-dimensional force sensors along the direction of gravity. Each force sensor can measure more than $10 \mathrm{~kg}$ weights, range of error was $\pm 0.1 \mathrm{~g}$, and the force data were shown on the screen. A mirror with the size of $150 \mathrm{~mm} \times 200 \mathrm{~mm}$ was fixed perpendicularly on the surface of the force sensors. The coordination $(X Y Z)$ is shown in Fig. 2. The dry adhesive material contacted with the mirror completely, and a ruler on the mirror was used to measure the moving distance and contact area. The direction of the peel-off force $(F)$ was downward, and had an angle $(\theta)$ with the gravity. The camera was used to record the angle $(\theta)$, peel-off tangential force $(F)$ and moving distance.

To measure the peel-off normal force of dry adhesive material, we carried out the experiments using the electronic scale which had four one-dimensional force sensors along the direction of gravity. Each force sensor can measure more than $10 \mathrm{~kg}$ weights, and the force data were shown on the screen. A mirror with the size of $150 \mathrm{~mm} \times 200 \mathrm{~mm}$ was fixed horizontally on the surface of the force sensors which were placed with more than $2,350 \mathrm{~g}$

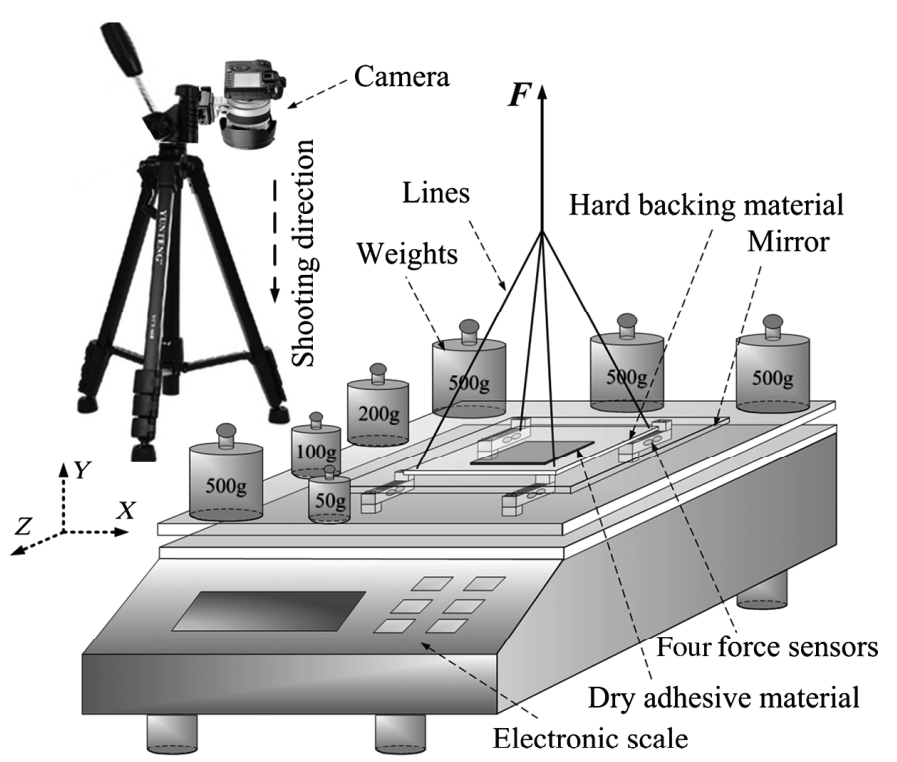

Figure 3. Equipment for the normal force testing.

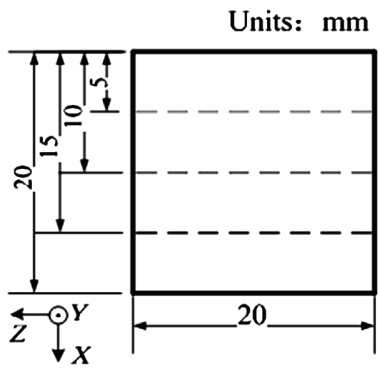

(a)

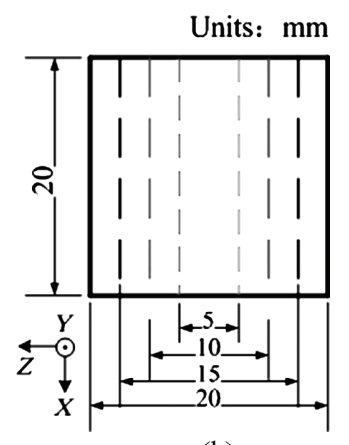

(b)
Figure 4. (a) Different contact areas for tangential force testing and (b) different contact areas for normal force testing.

as preload weights. The coordination $(X Y Z)$ is shown in Fig. 3. The dry adhesive material with the size of $20 \mathrm{~mm} \times 20 \mathrm{~mm}$ contacted with the mirror completely. The hard backing material tied down by four lines was fixed with another side of dry adhesive material. The direction of the peel-off normal force $(F)$ was upward, and opposite with the gravity. The camera was applied to take a video, and recorded the peel-off normal force $(F)$ and the changed contact area with different preloads.

\section{Testing Results}

A piece of dry adhesive material with $20 \mathrm{~mm} \times 20 \mathrm{~mm}$ size was selected in tangential force testing experiment. To analyse the relationship between the tangential force and contact area, the size of $20 \mathrm{~mm} \times 20 \mathrm{~mm}$ had been divided into four different areas.

As Fig. 4(a) shows, the length (a) of dry adhesive material is divided into four parts: $5,10,15$ and $20 \mathrm{~mm}$. As Fig. 2 shows, the angle $(\theta)$ was kept at zero. When the peel-off tangential force was increasing, the dry adhesive material will move along the direction of gravity slowly. 


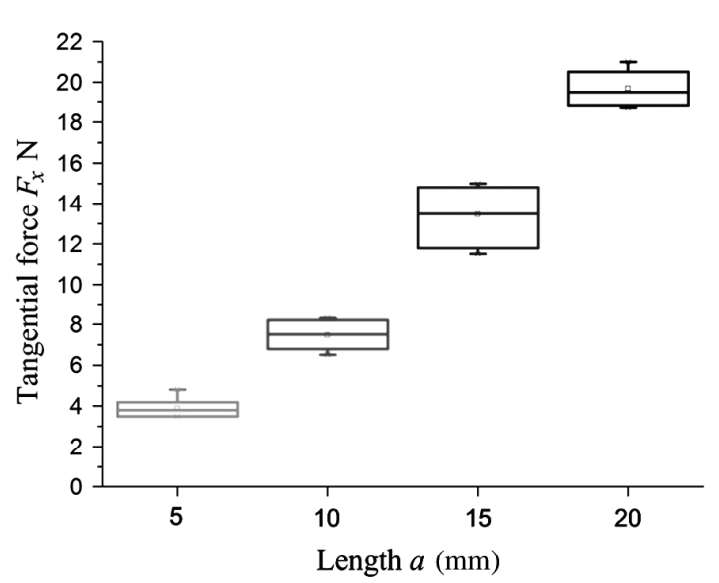

(a)

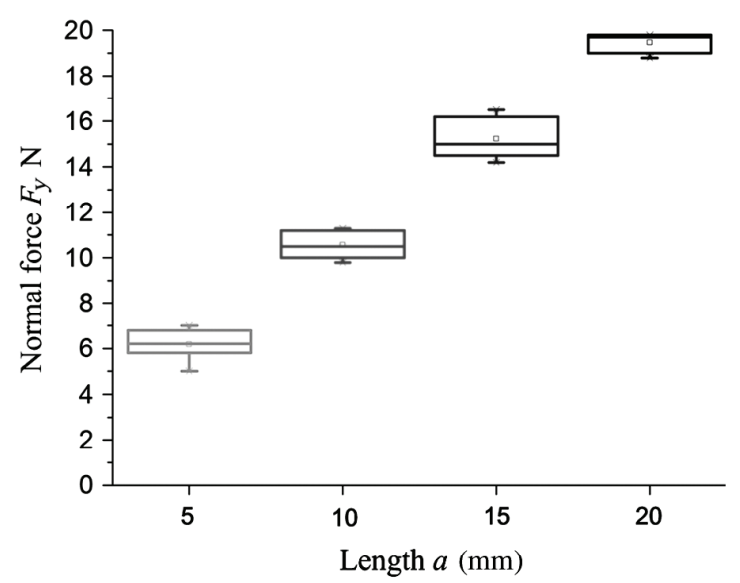

(b)

Figure 5. (a) Tangential forces testing with different contact areas and (b) normal forces testing with different contact areas.

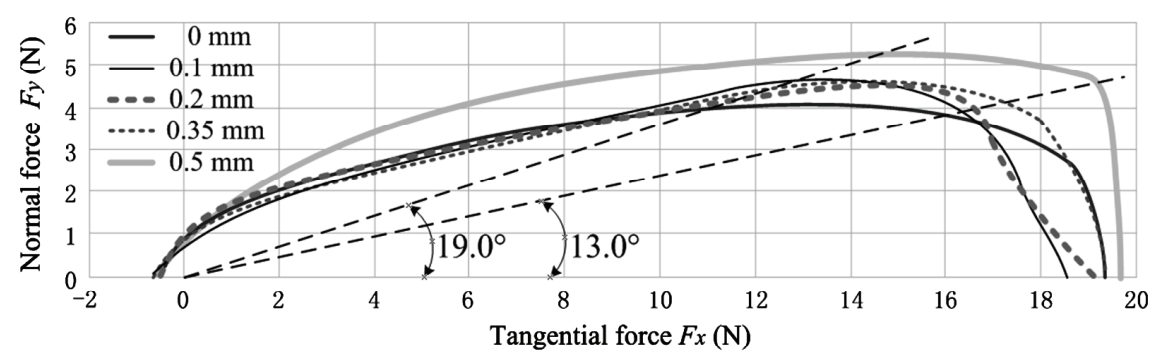

Figure 6. Peel-off force curves with different thickness substrate.

The average velocity of moving was about $1 \mathrm{~mm} / \mathrm{s}$. All the data will be tested more than seven times during the experiments. As the Fig. 5(a) shows, the average of the peel-off tangential force was about $19.4 \mathrm{~N}$ with length $a=20 \mathrm{~mm}$. The average of the peel-off tangential force was about $13.5 \mathrm{~N}$ with length $a=15 \mathrm{~mm}$. The average of the peel-off tangential force was about $7.5 \mathrm{~N}$ with length $a=10 \mathrm{~mm}$. The average of the peel-off tangential force was about $4.0 \mathrm{~N}$ with length $a=5 \mathrm{~mm}$. The linearity relationship between tangential force and contact area was obvious.

As Fig. 4(b) shows, the length (a) of dry adhesive material is divided into four parts: $5,10,15$ and $20 \mathrm{~mm}$. As Fig. 3 shows, the direction of the peel-off normal force $(F)$ was upward, and opposite with the gravity. When the peel-off normal force was increasing, the dry adhesive material will separate from the contact surface (mirror) quickly. All the data will be tested by more than seven times during the experiments. As the Fig. 5(b) shows, the average of the peel-off normal force was about $19.5 \mathrm{~N}$ with length $a=20 \mathrm{~mm}$. The average of the peel-off normal force was about $15.2 \mathrm{~N}$ with length $a=15 \mathrm{~mm}$. The average of the peel-off normal force was about $10.3 \mathrm{~N}$ with length $a=10 \mathrm{~mm}$. The average of the peel-off normal force was about $6.4 \mathrm{~N}$ with length $a=5 \mathrm{~mm}$. The linearity relationship between normal force and contact area was also obvious.

When we applied the dry adhesive material in gecko robot foot, the opposite side of dry adhesive material will attach with substrate of gecko robot foot. The different thickness substrate of gecko robot foot may be effect on the performance of adhesive force. Polyvinyl chloride (PVC) sheet had been applied in the substrate of gecko robot foot design. Four kinds of PVC with different thickness substrates were selected, such as $0.1,0.2,0.35$ and $0.5 \mathrm{~mm}$. However, one kind of the gecko robot foot was also designed using the dry adhesive material, and with no PVC sheet. Therefore, there were five kinds of gecko robot foot tested in the experiments.

To test the peel-off force curve, as Fig. 2 shows, the angle $(\theta)$ will change from $0^{\circ}$ to $180^{\circ}$. When the peeloff force was increasing, the dry adhesive material will be separated from contact surface (mirror) slowly. The electrical scale can measure the tangential force, and the camera can record the angle $(\theta)$ and tangential force $F_{x}$. Therefore, the normal force $F_{y}$ can calculate by angle $(\theta)$ and tangential force $F_{x}$, as below:

$$
F_{y}=F_{x} \cdot \tan (\theta)
$$

As Fig. 2 shows, the increasing peel-off force was forced with different directions, and the angle $(\theta)$ will change from $0^{\circ}$ to $180^{\circ}$. By recording the peel-off tangential force $F_{x}$ and the angle $(\theta)$, five peel-off force fitted curves had been calculated easily. As Fig. 6 shows, when the angle $(\theta)$ was changing from $0^{\circ}$ to $13.0^{\circ}$, the peel-off force, especially for tangential force, had a maximum value range; when the angle $(\theta)$ was from $13.0^{\circ}$ to $19.0^{\circ}$, the peel-off force, 


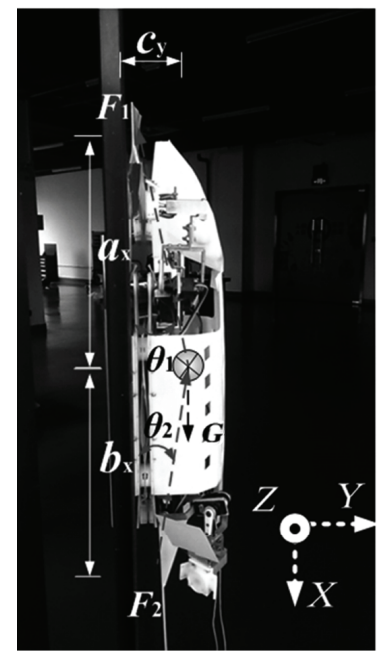

(a)

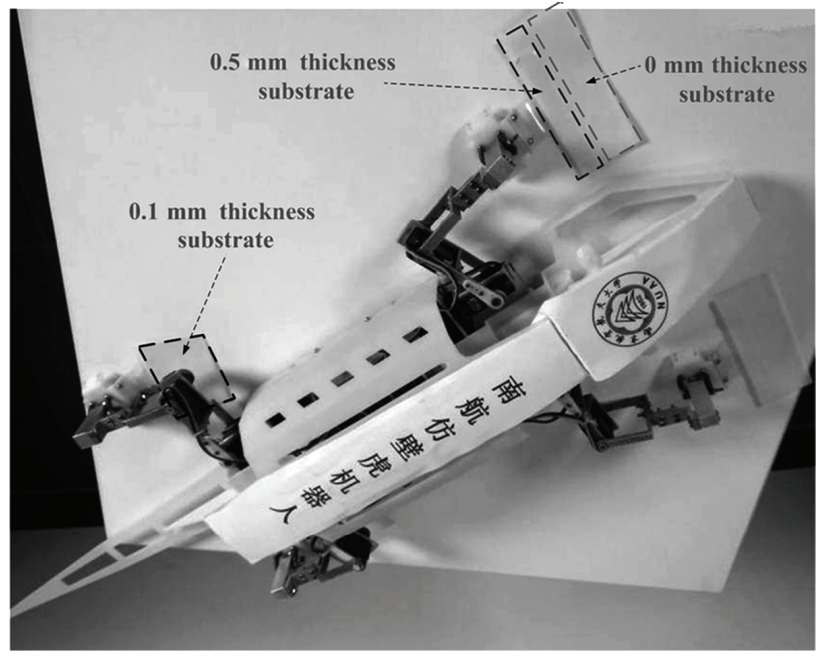

(b)

Figure 7. (a) The parameters of Gecko robot_6 when it was climbing vertically on the glass and (b) Gecko robot_6 with different thickness substrates on foot.

especially for normal force, had a maximum value range; when the angle $(\theta)$ was from $19.0^{\circ}$ to $180^{\circ}$, the peel-off force will reduce by increasing the angle $(\theta)$. But on the whole, the peel-off force with $0.5 \mathrm{~mm}$ thickness substrate was larger than the peel-off force with other thickness substrates $(0.35,0.2,0.1$ and $0 \mathrm{~mm})$. However, when the angle $(\theta)$ was about from $0^{\circ}$ to $10.0^{\circ}$, the peel-off force with $0 \mathrm{~mm}$ thickness was the second larger, and just less than the peel-off force with $0.5 \mathrm{~mm}$ thickness substrate. The peel-off force when the angle $(\theta)$ was from $90^{\circ}$ to $180^{\circ}$ was about one-fortieth of the peel-off force when the angle $(\theta)$ was about from $0^{\circ}$ to $13.0^{\circ}$.

\section{Discussion}

The testing results had been shown, and how can we apply these data to design the gecko robot foot and guide the locomotion control was a challenging project. When the gecko robot was climbing the wall using dry adhesive material, the adhesive force will support all its weights. The adhesive force was not just using single normal force or tangential force, but normal force and tangential force together. Therefore, the peel-off force curve was very important key factor for application of dry adhesive material. During the experiments, the big thickness substrate of gecko robot foot contact with the mirror well by big preload, and its adaptability with the flat surface was not very well. However, gecko robot foot with $0 \mathrm{~mm}$ thickness substrate can contact with the mirror well by little preload, and its adaptability with the flat surface was very well.

The parameters of Gecko robot_6 is shown in Fig. 7 (a). The gravity of Gecko robot_ $\overline{6}$ was parameter $G$. The distance between front foot and centre of gravity was parameter $a_{x}$ along the axis $X$, and the distance between rear foot and centre of gravity was parameter $b_{x}$ along the axis $X$. The distance between centre of gravity and glass was parameter $c_{y}$ along the axis $Y . F_{1}$ and $F_{2}$ which had the angles $\theta_{1}$ and $\theta_{2}$ with glass were adhesive forces from the front and rear foot. The relationships of these parameters are given below:

$$
\begin{gathered}
\left\{\begin{array}{c}
F_{1} \sin \left(\theta_{1}\right)=F_{2} \sin \left(\theta_{2}\right) \\
F_{1} \cos \left(\theta_{1}\right)+F_{2} \cos \left(\theta_{2}\right)=G
\end{array}\right. \\
\left\{\begin{array}{l}
\theta_{1}=\arctan \left(c_{y} / a_{x}\right) \\
\theta_{2}=\arctan \left(c_{y} / b_{x}\right)
\end{array}\right.
\end{gathered}
$$

As Fig. 7(b) shows, Gecko robot_6 was designed with four legs. The front foot was applied with dry adhesive material with $0 \mathrm{~mm}$ thickness substrate and $0.5 \mathrm{~mm}$ thickness substrate, and the rear foot was applied with dry adhesive material with $0.1 \mathrm{~mm}$ thickness substrate.

The $0 \mathrm{~mm}$ thickness substrate on front foot will help the gecko robot contact with glass well using little preload, $0.5 \mathrm{~mm}$ thickness substrate will increase the normal force $F_{1} \sin \left(\theta_{1}\right)$ and adhere to the glass stably. However, when rear foot contact with the glass, there was preload $F_{2} \sin \left(\theta_{2}\right)$ which help the rear foot with $0.1 \mathrm{~mm}$ thickness substrate contact with the glass well, and it was peeled off the glass easily because there was minimum peeloff force during the angle from $90^{\circ}$ to $180^{\circ}$. In general, the selections of gecko robot foot design were depended on these factors, such as the adaptability and peel-off force, adhesive force. How to keep these factors balance during the gecko robot locomotion will be very important for gecko robot.

\section{Experiment and Results}

The climbing experiment of Gecko robot 6 which had about $900 \mathrm{~g}$ weights, the sizes of $500 \mathrm{~mm} \times 300 \mathrm{~mm} \times$ $100 \mathrm{~mm}$, and 12 degrees of freedom was carried out on a vertical flat glass $\left(90^{\circ}\right)$. As Fig. 8 shows, the successful 

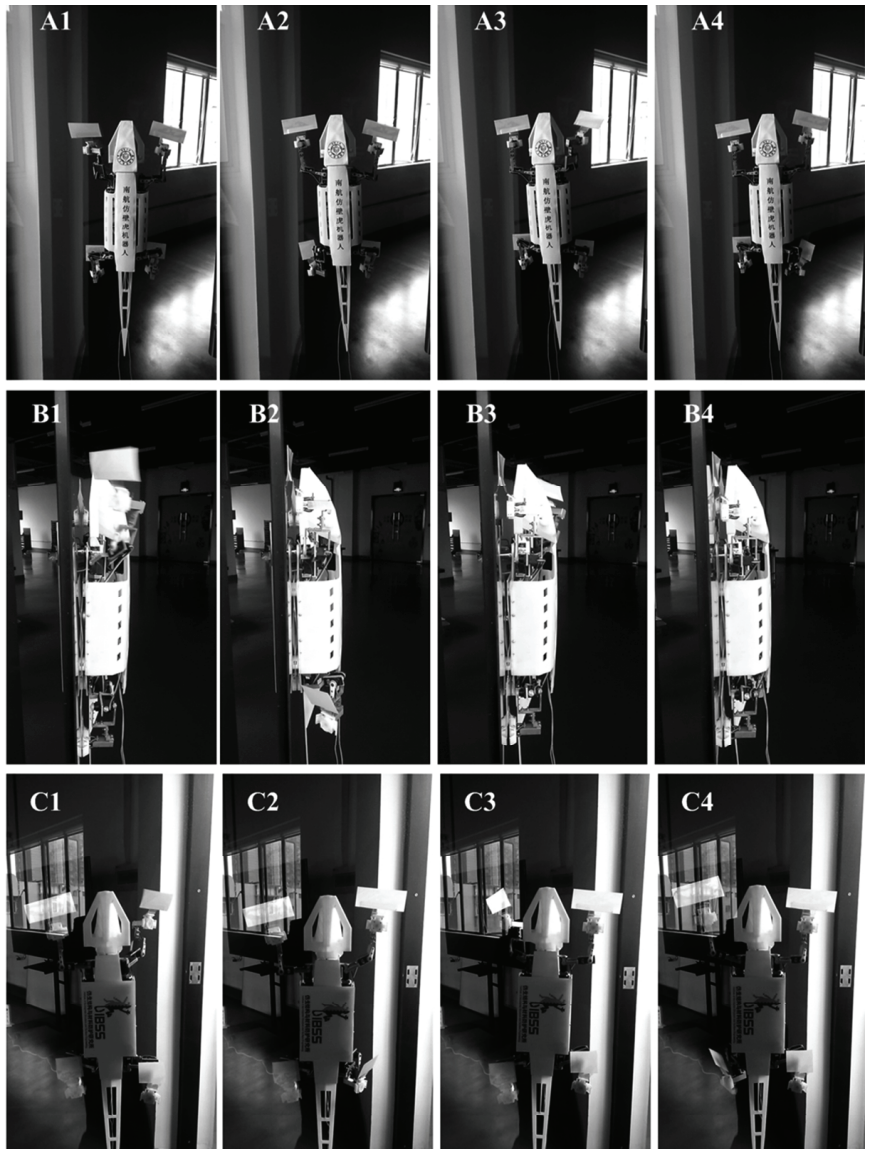

Figure 8. The climbing locomotion of Gecko robot_6 on a vertical glass.

climbing locomotion was stable. The front foot contacted with the glass well and rear foot peeled off the glass surface easily. The angle $\theta_{1}$ measured using the camera was about $14.4^{\circ} \pm 2.0^{\circ}$, and it was changed around $13^{\circ}$ and was also in the applied range of $0^{\circ}-19^{\circ}$.

\section{Conclusion}

One kind of the dry adhesive materials with MSAMS was selected and firstly applied in vertical locomotion of Gecko robot_6 which was a bio-inspired gecko robot with four legs. The adhesive ability of MSAMS was tested, and the new bio-inspired gecko foot with MSAMS was designed. The Gecko robot_6 can climb on a smooth vertical surface (glass) stably using dry adhesive material with MSAMS. The experiments show that the application of MSAMS in Gecko robot 6 is successful. In the future, the locomotion experiments of Gecko robot_ 6 on the ceiling will be carried out by using dry adhesive material, and the adhesive property of dry adhesive material will be tested for further study and application. Adhesive model will be discussed in future research work.

\section{Acknowledgement}

This work was supported by the National Natural Science Foundation of China (Grant Nos. 51475230, 51105201, 51435008). We thank Gorb for providing the previous dry adhesive material testing data and some important papers. All reviewers are thanked for their constructive advises.

\section{References}

[1] K. Autumn, Y.A. Liang, S.T. Hsieh, et al., Adhesive force of a single gecko foot-hair, Nature, 405, 2000, 681-685.

[2] K. Autumn and A.M. Peattie, Mechanisms of adhesion in geckos, Integrative and Comparative Biology, 42, 2002, 10811090.

[3] K. Autumn, How gecko toes stick: The powerful, fantastic adhesive used by geckos is made of nanoscale hairs that engage tiny forces, inspiring envy among human imitators, American Scientist, 94, 2006, 124-132.

[4] K. Autumn, M. Sitti, A. Peattie, et al., Evidence for van der Waals adhesion in gecko setae, Proceedings of the National Academy of Sciences of the United States of America, 99(19), 2002, 12252-12256.

[5] K. Autumn, S.T. Hsieh, D.M. Dudek, et al., Dynamics of geckos running vertically, Journal of Experimental Biology, 209(2), 2006, 260-272.

[6] Z. Dai, Z. Wang, and A. Ji, Dynamics of gecko locomotion: A force-measuring array to measure $3 \mathrm{D}$ reaction forces, Journal of Experimental Biology, 214(5), 2011, 703-708.

[7] Z. Wang, W. Gu, Q. Wu, and Z. Dai, Morphology and reaction force of toes of geckos freely moving on ceilings and walls, Science China Technological Sciences, 53(6), 2010, 1688-1693.

[8] Z. Wang, Z. Dai, A. Ji, et al., Biomechanics of gecko locomotion: the patterns of reaction forces on inverted, vertical and horizontal substrates, Bioinspiration \& Biomimetics, 10(1), 2015, 1-14

[9] M. Sitti and R.S. Fearing, Synthetic gecko foot-hair micro/nano-structures for future wall-climbing robots, Proc. IEEE Int. Conf. on Robotics $\&$ Automation, Taipei, Taiwan, 2003, 1164-1170.

[10] B. Aksak, M.P. Murphy, and M. Sitti, Gecko inspired microfibrillar adhesives for wall climbing robots on micro/nanoscale rough surfaces, IEEE Int. Conf. Robotics and Automation, Pasadena, CA, 2008, 3058-3063.

[11] S. Das, N. Cadirov, S. Chary, Y. Kaufman, et al., Stick-slip friction of gecko-mimetic flaps on smooth and rough surfaces, Journal of the Royal Society Interface, 12(104), 2015, 1-9.

[12] K.A. Daltorio, S.N. Gorb, A. Peressadko, A.D. Horchler, et al., A robot that climbs walls using micro-structured polymer feet, in M.O. Tokhi (ed.), Climbing and Walking Robots, (Berlin Heidelberg: Springer-Verlag, 2006), 131-138.

[13] X. Wu, D. Wang, A. Zhao, D. Li, et al., A wall-climbing robot with biomimetic adhesive pedrail, in D. Zhang (ed.), Advanced Mechatronics and MEMS Devices, 9 (New York: Springer-Verlag, 2013), 179-191.

[14] T. Seo and M. Sitti, Tank-like module-based climbing robot using passive compliant joints, IEEE/ASME Transactions on Mechatronics, 18(1), 2013, 397-408.

[15] K.H. Koh, M. Sreekumar, and S.G. Ponnambalam, Hybrid electrostatic and elastomer adhesion mechanism for wall climbing robot, Mechatronics, 35, 2016, 122-135.

[16] C. Menon, M. Murphy, and M. Sitti, Gecko inspired surface climbing robots, Proc. IEEE Int. Conf. Robotics and Biomimetics, Shenyang, China, 2004, 431-436.

[17] C. Menon and M. Sitti, A biomimetic climbing robot based on the gecko, Journal of Bionic Engineering, 3(3), 2006, 115-125.

[18] O. Unver, A. Uneri, A. Aydemir, and M. Sitti, Geckobot: A gecko inspired climbing robot using elastomer adhesives, Proc. IEEE Int. Conf. Robotics and Automation, Orlando, FL, 2006, 2329-2335.

[19] B. He, Z. Wang, M. Li, et al., Wet adhesion inspired bionic climbing robot, IEEE/ASME Transactions on Mechatronics, 19(1), 2014, 312-320.

[20] R. Chen, R. Liu, J. Chen, and J. Zhang, A gecko inspired wall-climbing robot based on electrostatic adhesion mechanism, Proc. IEEE Int. Conf. Robotics and Biomimetics, Shenzhen, China, 2013, 396-401.

[21] P. Birkmeyer, A.G. Gillies, and R.S. Fearing, Dynamic climbing of near-vertical smooth surfaces, IEEE/RSJ Int. Conf. 
Intelligent Robots and Systems, Vilamoura, Algarve, Portugal, 2012, 286-292.

[22] R. Yue, J. Xiao, K. Li, et al., Design and performance analysis of retractable-claw wheels for field robots, International Journal of Robotics and Automation, 25(3), 2010, 250-258.

[23] M.A.K. Jaradat, S.M. Ashour, A.A. Matalkh, et al., Biologically inspired design of a glass climbing robot for remote services, International Journal of Robotics and Automation, 25(2), 2010, 132-141.

[24] M. Henrey, J. Krahn, A. Ahmed, K. Wormnes, et. al., Climbing with structured dry adhesives: sticky robots for scaling smooth vertical surfaces, the 12th Symposium on Advanced Space Technologies in Robotics and Automation, Sydney, Australia, 2013, 1-6.

[25] W. Wang, S. Wu, P. Zhu, and R. Liu, Analysis on the dynamic climbing forces of a gecko inspired, climbing robot based on GPL model, IEEE/RSJ Int. Conf. Intelligent Robots and Systems, Hamburg, Germany, 2015, 3314-3319.

[26] S. Kim, M. Spenko, S. Trujillo, et al., Whole body adhesion: Hierarchical, directional and distributed control of adhesive forces for a climbing robot, IEEE Int. Conf. Robotics and Automation, Roma, Italy, 2007, 1268-1273.

[27] S. Nansai and R.E. Mohan, A survey of wall climbing robots: Recent advances and challenges, Robotics, 5(3), 2016, 1-14.

[28] A. Zhu and S.X. Yang, Tracking control of a mobile robot with stability analysis, International Journal of Robotics and Automation, 28(4), 2013, 340-348.

[29] S.X. Yang, A. Zhu, M.Q.H. Meng, et al., A bioinspired neurodynamics-based approach to tracking control of mobile robots, IEEE Transactions on Industrial Electronics, 59(8), 2012, 3211-3220.

[30] S. Gorb, M. Varenberg, A. Peressadko, and J. Tuma, Biomimetic mushroom-shaped fibrillar adhesive microstructure, Journal of the Royal Society Interface, 4(13), 2007, $271-275$.

[31] A.E. Kovalev, M. Varenberg, and S.N. Gorb, Wet versus dry adhesion of biomimetic mushroom-shaped microstructures, Soft Matter, 8(29), 2012, 7560-7566.

[32] L. Heepe and S.N. Gorb, Biologically inspired mushroomshaped adhesive microstructures, Annual Review of Materials Research, 44, 2014, 173-203.

\section{Biographies}

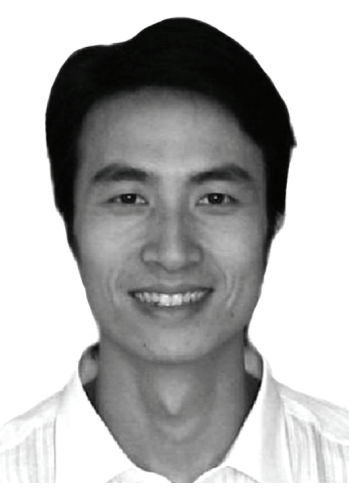

Zhiwei $Y u$ received his Ph.D. degree in the College of Mechanical and Electrical Engineering from Harbin Engineering University, Harbin, China, in 2008. Currently, he is an Associate Professor of College of Astronautics at Nanjing University of Aeronautics and Astronautics, Nanjing, China. He was a Visiting Professor in the Advanced Robotics \& Intelligent Systems (ARIS) Laboratory at the University of Guelph in Canada from December 2014 to January 2016. He has published over 20 papers in related international conferences and journals. His research interests include bio-inspired robots, legged robots, locomotion control and intelligent systems.

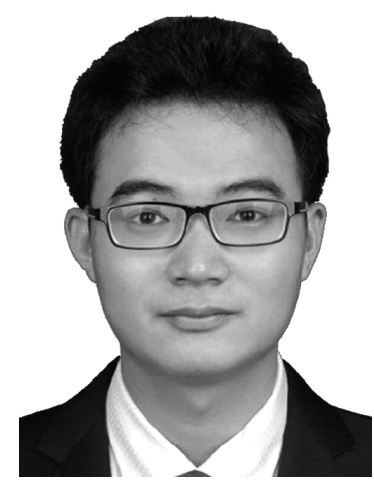

Bin Yang received his bachelor's degree in engineering from Shenyang Ligong University, Shenyang, China, in 2012. Currently, he is a postgraduate in the College of Astronautics at Nanjing University of Aeronautics and Astronautics, Nanjing, China. His research interests include gecko robot and locomotion control.

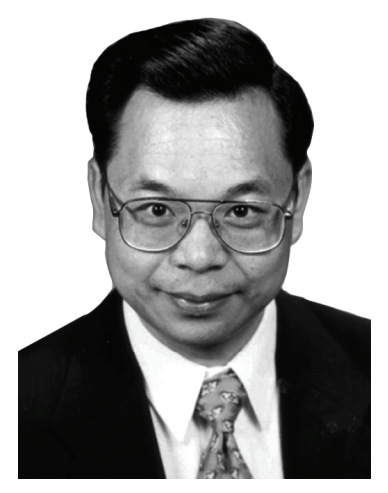

Simon $X$. Yang received the B.Sc. degree in engineering physics from Beijing University, China in 1987, the first of two M.Sc. degrees in biophysics from Chinese Academy of Sciences, Beijing, China, in 1990, the second M.Sc. degree in electrical engineering from the University of Houston, USA, in 1996, and the Ph.D. degree in electrical and computer engineering from the University of Alberta, Edmonton, Canada in 1999. He joined the School of Engineering at the University of Guelph, Canada, in 1999. Currently, he is a Professor and the Head of the Advanced Robotics \& Intelligent Systems (ARIS) Laboratory at the University of Guelph in Canada. His research interests include intelligent systems, robotics, sensors and multi-sensor fusion, wireless sensor networks, control systems, soft computing and computational neuroscience.

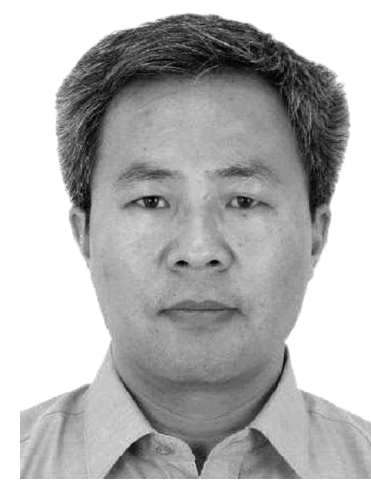

Zhendong Dai received his Ph.D. degree in the College of Mechanical and Electrical Engineering from Nanjing University of Aeronautics and Astronautics, Nanjing, China, in 2000. Currently, he is a Professor and the Head of Institute of Bio-inspired Structure and Surface Engineering (IBSS) at Nanjing University of Aeronautics and Astronautics, Nanjing, China. He has published over 200 papers in related international conferences and journals. His research interests include bio-inspired systems, robotics, bionic structure and material, multi-sensors, tribology and neurobiology. 